\title{
Calibration trending in the Spitzer beyond era
}

Patrick J. Lowrance, Jessica E. Krick, Jim G. Ingalls, Seppo Laine, Sean J. Carey, et al.

Patrick J. Lowrance, Jessica E. Krick, Jim G. Ingalls, Seppo Laine, Sean J. Carey, William Glaccum, Joseph L. Hora, Carl Grillmair, "Calibration trending in the Spitzer beyond era," Proc. SPIE 10704, Observatory Operations:

Strategies, Processes, and Systems VII, 1070427 (10 July 2018); doi: $10.1117 / 12.2313953$

Event: SPIE Astronomical Telescopes + Instrumentation, 2018, Austin, Texas, United States 


\title{
Calibration Trending in the Spitzer Beyond Era
}

\author{
Patrick J. Lowrance*a, Jessica E. Krick ${ }^{\text {a }}$, Jim G. Ingalls a , Seppo Laine ${ }^{\text {a }}$, Sean J. Carey ${ }^{\text {a }}$, William \\ Glaccum $^{\text {a }}$, Joseph L. Hora ${ }^{\mathrm{b}}$, Carl J. Grillmair ${ }^{\mathrm{a}}$ \\ a IPAC, M/S 314-6, California Institute of Technology, 1200 E. California Blvd., Pasadena, CA, \\ 91125, USA; ${ }^{\mathrm{b}}$ Harvard-Smithsonian Center for Astrophysics, Cambridge, MA USA 00555-9642
}

\begin{abstract}
The Spitzer Space Telescope currently operates in the "Beyond Era", over nine years past an original cryogenic mission. As the astronomy community continues to advance scientific boundaries and push beyond original specifications, the stability of the Infrared Array Camera (IRAC) instrument is paramount. The Instrument Team (IST) monitors the pointing accuracy, temperature, and calibration and provides the information in a timely manner to observers. The IRAC IST created a calibration trending web page, available to the general astronomy community, where the team posts updates of three most pertinent scientific stability measures of the IRAC data: calibration, bias, and bad pixels. In addition, photometry and telescope properties from all the staring observations ( $>1500$ as of April 2018) are trended to examine correlations with changes in the age or thermal properties of the telescope. A long, well-sampled baseline established by consistent monitoring outside anomalies and space weather events allows even the smallest changes to be detected.
\end{abstract}

Keywords: infrared instrumentation, calibration, space mission;

\section{INTRODUCTION}

NASA's Spitzer Space Telescope ${ }^{1}$, one of NASA's Great Observatories, continues to operate past the original cryogenic mission concept (2003-2009), executing both a follow-on "Warm" mission (2009-2016) and now into the current "Beyond" (2016-present) mission phase. As Spitzer closes out its fifteenth year of operations, the mission continues to operate well beyond its initial five-year primary mission due to several optimizations used to overcome challenges. In 2016, another 2.5-year final extension was awarded with a recent additional 8 months to extend Spitzer operations through November 2019. This extension would have allowed Spitzer operations to overlap with the James Webb Space Telescope, planned at that time to launch in early 2019. Within this "Beyond" phase, the astronomical community continues to advance scientific boundaries from exploring relatively nearby exoplanets to the farthest galaxies. As Spitzer is pushed beyond its original specifications, the understanding of the stability of the Infrared Array Camera ${ }^{2}$ (IRAC) instrument is paramount.

As the age and orbit of the Spitzer Space Telescope (Figure 1) evolves, the IRAC Instrument Support Team (IST) continuously monitors the calibration, bias levels, number of radiation hits, and space weather anomalies. This information is continually updated and provided to observers. A calibration trending web page ${ }^{3}$ was created, available to the general astronomy community, where the team posts roughly biweekly updates on stability measures of the IRAC data.

Spitzer/IRAC has a unique calibration program. ${ }^{4,5}$ In order to convert measured flux on the detector into physical units, IRAC has a 2 stage calibration star program. The primary calibration stars are used to derive the absolute calibration, done separately for the cryogenic and the warm mission. The secondary calibration stars are used to check on a regular (daily) basis that the absolute calibration has not changed. This is critical to science operations because the Spitzer observations need to be put into physical units to be compared with other ground and space based facility measurements. These calibration stars consist of $\mathrm{A}$ and $\mathrm{K}$ stars chosen carefully to be devoid of astrophysical variations. The calibration program provides absolute photometric uncertainties less than $3 \%$.

In addition to absolute photometric calibration, the IRAC IST designed a dark calibration program for shutterless operation. In conventional dark calibration programs, the shutter would be closed to block light from the telescope at which time the electronic level of the bias is measured. Because IRAC does not close it's shutter, instead, a relatively

Observatory Operations: Strategies, Processes, and Systems VII, edited by Alison B. Peck,

Robert L. Seaman, Chris R. Benn, Proc. of SPIE Vol. 10704, 1070427 · C 2018 SPIE

CCC code: $0277-786 \mathrm{X} / 18 / \$ 18 \cdot$ doi: $10.1117 / 12.2313953$ 
"dark" field is observed weekly in the full suite of exposure times to measure both an overall level of the bias and to monitor any changes in that level. The dark calibration field is located very close to the north ecliptic pole, which has the fewest infrared bright stars possible.

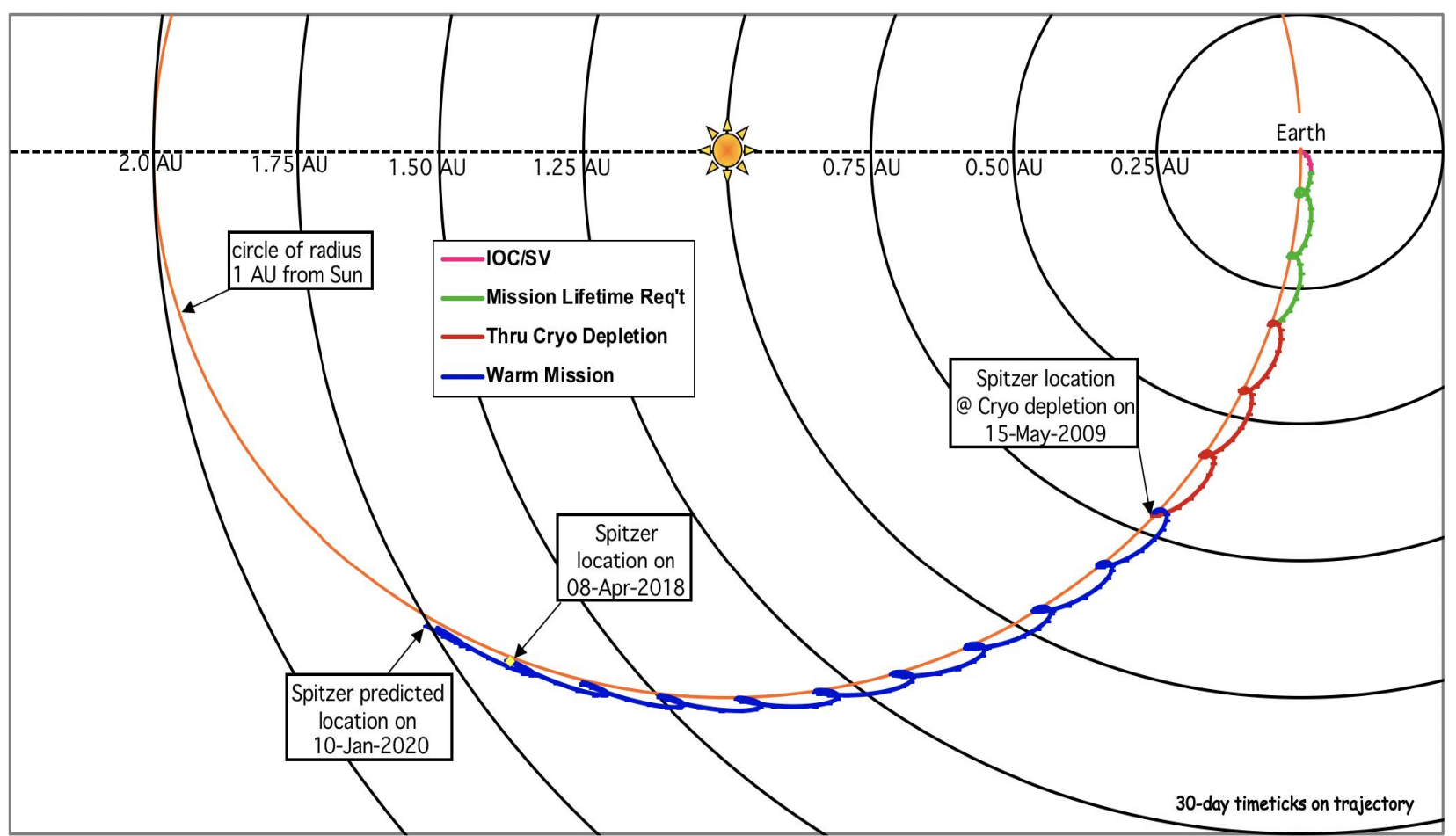

Figure 1: Spitzer's Earth-trailing Solar Orbit, projected onto the Ecliptic plane. In the rotating coordinate frame shown here, the Earth-Sun line (x-axis) is held fixed and the Earth is at the origin. Distance contours shown are in increments of $0.25 \mathrm{AU}$.

The final monitored topic for the calibration trending website is radiation hits. Extragalactic and Solar cosmic rays will temporarily saturate the pixel they impact. This effectively reduces the number of pixels available for science, and so the number of impacted pixels is monitored. An average of 5 pixels per second will still only effect $0.5 \%$ of pixels in a full array 100 second exposure (IRAC's longest allowable exposure time), so this number currently does not have a negative effect on science. As further discussed in Section 3, solar activity can emit a shower of radiation above an energy threshold that will affect the IRAC arrays by saturating over 100 pixels per second. This will overwhelm the astronomical observations and render the data unusable. These type of events have occurred less than six times in the mission, and the observations are typically rescheduled for a later observation.

Both the characterization of exoplanet atmospheres and monitoring of the physics of the super-massive black hole at the center of our Galaxy have been possible due to time series observations using IRAC over long time periods (hours to days). These time series observations observe temporal variations in relative photometry down to less than $0.001 \%$. Since observers quickly discovered the main systematic limiting the high precision photometry is small telescope pointing oscillations coupled with intra-pixel sensitivity variations, the IST worked to understand and mitigate these effects. ${ }^{6,7}$ As motions of a target across a pixel can be correlated with changes in the thermal properties of the telescope ${ }^{8}$, the IST has made operational changes, taken specialized calibration data, and provided suggestions for best practices for observation planning and design. To understand if the quality of science over the mission remains constant, the IST has been trending all the staring observations ( $>1500$; as of Apr 2018) for correlations of any changes within observations. 
Consistent monitoring and communication with observers outside anomalies and space weather events is significant. The observers want to understand that even the smallest changes in the stability of the instrument that can lead to possible negative effect are being monitored.

\section{CALIBRATIONS TRENDS}

The calibration trending web page, available to the general astronomy community, was created at the end of 2015 in order to post biweekly measures of three most pertinent scientific stability measures of the IRAC data. The first is the stability of the instrument response seen through the calibration stars. The same set of primary calibration stars are observed once a week, and the normalized flux densities for both channel 1 (3.6um) and channel 2 (4.5um) are plotted. The second metric is the stability of the bias offset measured in the sky darks. A "sky dark" frame is created from observations of a low stellar density and zodiacal background region and subtracted from observations as a calibration. The median values are examined and plotted as a time series to monitor the stability. Finally, the number of total pixels affected by all energetic particles or cosmic rays is tracked every week. During the mission, the statistical baseline of average affected pixels per second for each detector remains at 4-6 pixels per second in the absence of a solar event. All of the metrics are stable well within specifications outside of any solar events or anomalies. The figures in this section all come from the current webpage.

\subsection{Photometric Stability}

The IRAC IST monitors the $3.6 \mu \mathrm{m}$ and $4.5 \mu \mathrm{m}$ calibration star normalized flux densities by periodically checking that the aperture photometry of the calibration stars remains nominal throughout the mission. The calibration stars include twenty-one primary and secondary stars chosen at the beginning of the cryogenic mission. Primary calibrators are located in the continuous viewing zone so the entire set of primary stars can be observed every two weeks. The set of secondary calibrators have positions near the ecliptic plane and therefore only one is visible at any given time. A secondary calibrator is observed roughly daily for a few weeks before switching to the next star in the set. In Figure 2, the primary calibrators are shown in blue, red, black, and green colors; the secondary calibrators are shown with a multitude of repeating colors as they cycle through the year. The primary calibrator data points are mostly not visible behind the more frequently observed secondary calibrator data points (but we will see them if they move away from the linear trend).
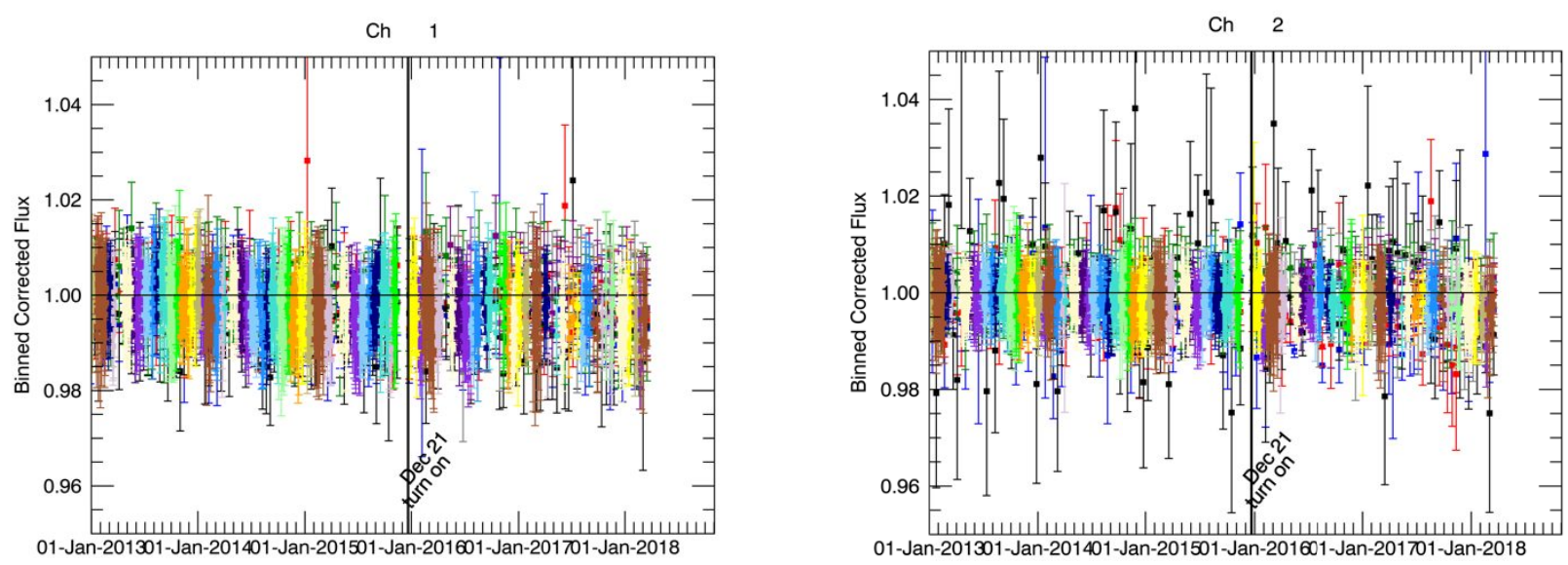

Figure 2. The $3.6 \mu \mathrm{m}$ (left) and 4.5 (right) $\mu \mathrm{m}$ calibration star normalized flux densities as a function of time from 2013 to the present. All flux densities are measured by aperture photometry and include 21 primary and secondary calibrators denoted with different colors. The line (and gap) at Dec 2015 denotes an anomaly in which IRAC was off and no observations were taken. There is no difference seen before or after this anomaly. 
The flux densities have been measured using aperture photometry corrected for both the location of the center of the star with respect to the pixel (pixel phase effect) and the location on the array (array location dependence). The flux densities for each star are normalized to the median value for that star. Error bars are calculated as the sigma clipped standard deviation in the bins divided by the square root of the number of data points in each bin. The black line at normalized flux density $=1.0$ is shown to aid the eye in determining what a flat line would look like. All data have been processed with pipeline version S19.2. These 21 stars include full array and subarray observations, as well as many different exposure times and well depths. These plots are updated weekly as new observations are made. The gap at December 2015 is from an anomaly, with no obvious effect on the calibration after the instrument was turned back on.

Over the course of the entire mission, we do see a decrease in sensitivity of IRAC photometry of order $0.1 \%$ per year in channel 1 and $0.05 \%$ per year in channel 2 . The suspected cause is radiation damage to the optics, which can be expected in the space environment.

\subsection{Bias Stability}

Instead of using a shutter, dark current and bias offsets are calibrated by observing a dark region of the sky ("skydark") near the north ecliptic pole every seven days. The resulting image contains both the instrumental bias and dark current as well as sky background level which is dominated by zodiacal light. ${ }^{9}$ Models of zodiacal light contributions are used to remove that signature, leaving just an image of the instrumental signatures. The instrumental signatures can then be subtracted from the routine science data, leaving only astrophysical sources. To monitor for any changes in the bias, the IST routinely examines the median value of the skydarks after they have been pipeline-processed. The calibration skydarks are created in a dedicated pipeline in a similar manner to that of the science data. A median ensemble skydark image is created from a dithered suite of eighteen individual images using an outlier rejection and sophisticated spatial filtering to reject astronomical objects in the frames. A skydark is created for every frametime once a week and the nearest-in-time skydark is used for image calibration. While all frametimes have bias levels monitored, similar trends in the background value as a function of time over the mission are seen in all frametimes, so only one frametime is currently used for monitoring. The $12 \mathrm{~s}$ frame time was chosen for its balance between read noise and background noise so that changes in the bias level can be seen most clearly.

For every combined $12 \mathrm{~s}$ skydark frame, a median value of the array is calculated and plotted with error bars over the course of the warm mission (Figure 3). Error bars are calculated from the Poisson electron noise and readnoise error added in quadrature calculated in the processing. The red line traces out the predicted zodiacal variation for each wavelength band normalized to the median value of the skydark in Jan 2012 to discern it from any change in the skydarks. There does appear to be a slight trend $(<0.1 \% /$ year $)$ of decreasing bias in both channel 1 and 2 . This could be due to a decrease in zodiacal background as the distance of the spacecraft has increased from Earth, or could be due in part to a decrease in the sensitivity of the optics as discussed in the photometry. It is hard to discern either from the data. Any change in bias in the skydark frames will also appear in the science frames, so it is subtracted out and does not cause any change in the overall calibration of the instrument, but the IST continues to monitor it. 

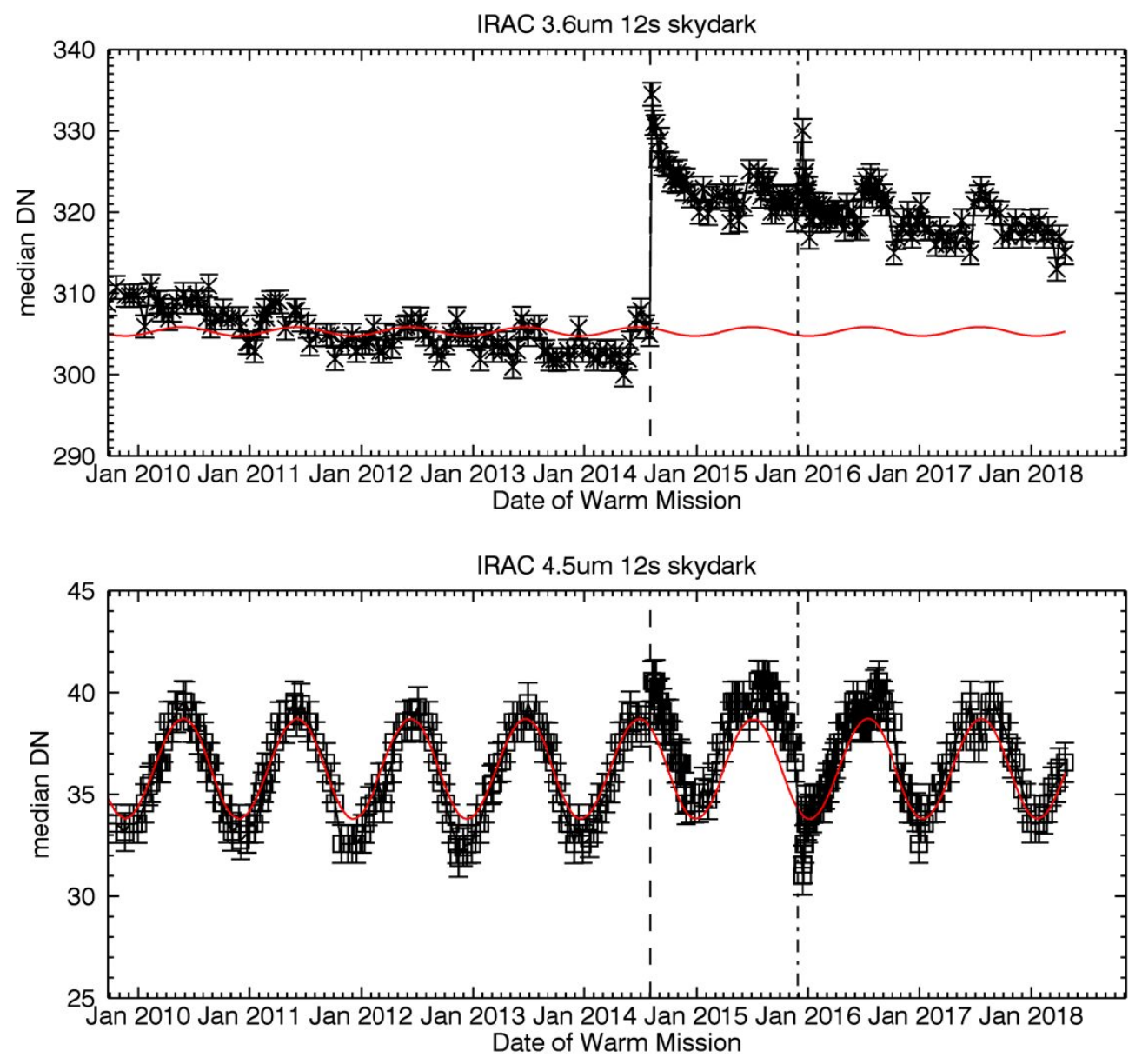

Figure 3. Medan value of the 12s skydarks plotted over time since beginning of the Warm Mission. The vertical dotted lines denote two anomalies in which the array was reset. The median value was observed to return to a "new" nominal level. The red lines are the shape of the predicted zodiacal variation normalized to the median value of skydarks in Jan 2012 to discern from any trending change in skydark values.

The two vertical lines in the plot denote two anomalies, which occurred on 2014-08-02 and 2015-11-28. The first one caused the arrays to reset with less than the usual applied reverse voltage across the detectors. After normal operation was restored, the dark bias levels settled back to level $3.2 \%$ higher in both channels. The second event caused IRAC to turn off, or "safe". After recovery, the $3.6 \mu \mathrm{m}$ array quickly settled back to the same higher bias, however the temperature of the $4.5 \mu \mathrm{m}$ array was below nominal for over a week while off. Once its normal temperature was restored, the dark bias quickly settled to level slightly lower than it was between the anomalies.

\subsection{Radiation Hits}

Extra-solar high energy particles and cosmic rays are the primary sources of radiation hits on IRAC. The number of affected pixels is tracked using the calibration observations taken with IRAC once a week. During the mission, the statistical baseline of the average number of affected pixels per second has been established as approximately 4-6 saturated pixels per second (see Figure 4). The trend observed in IRAC data follows the inverse of the solar cycle, as expected, with less cosmic rays during the peak of the solar activity. One possible explanation that the cosmic ray intensity is out of phase is that the magnetic fields from the solar wind sweep away cosmic rays. ${ }^{10}$ 
The number of cosmic rays affecting an IRAC image is determined from the calibration skydark measurements of 100s frametimes taken once a week, using the same techniques as were preformed in the cryogenic mission. ${ }^{11}$ The dark frames consist of 27 individual images taken in a $3 \times 3$ mapping pattern in the "Best NEP dark" region chosen at the beginning of the mission. This is within the continuous viewing zone and contained no bright sources that would saturate in $100 \mathrm{~s}$ images. A mosaic image is created from the individual frames of the dark field using outlier rejection to remove transients. The individual images are registered and compared with the mosaic to determine the affected pixels per second using the number of pixels above a cutoff level (after masking brighter stars in the image). The pipeline also has an outlier rejection which removes transient pixels and registers them in a mask file. The number of counts in this mask file can be compared and match the cosmic ray statistics.

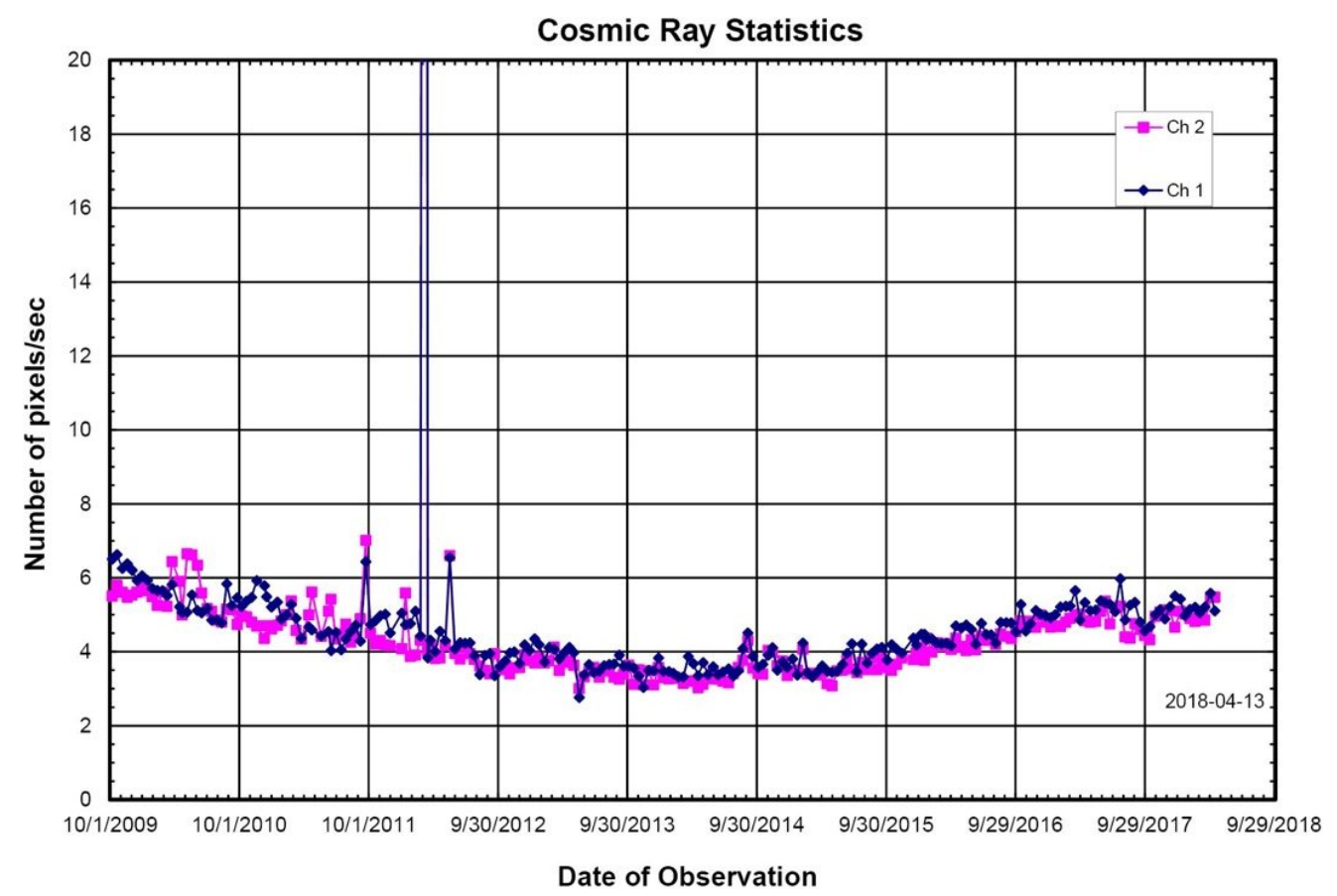

Figure 4. Average number of pixels per second affected by cosmic rays in the calibration 100s skydarks since the beginning of the warm mission. The spike in 2012 is due to a solar flare that occurred during the time the dark calibrations were being taken.

Further discussed in Section 3, the IRAC IST actively monitors solar coronal mass ejection (CME) events that can sometimes raise the number of affected pixels by a factor of 10-15 due to the large number of high energy particles produced. While IRAC images are always constantly monitored, the IST performs even more careful checks of image quality for data taken during several days around the predicted impact time of a CME event on the spacecraft to determine whether any observations need to be retaken.

\section{IMPACTS OF SOLAR FLARES}

A solar flare is a rapid release of energy in the Sun's corona and can produce energy across the electromagnetic spectrum. A coronal mass ejection (CME), usually the consequence of a solar flare $\mathrm{C}$, are high energy particles that travel away from the solar surface. As the relationship between flares and CME's are still an area of active research, the terms "flare" and "CME" are used interchangeably in considering the space weather effects on spacecraft.

Spitzer was designed with radiation-hardened shielding and tested in an environment based on the original five-year mission lifetime. It was thought that the instruments might be susceptible to radiation damage from a strong space weather event producing protons with energy levels exceeding $100 \mathrm{MeV}$ and particle flux greater than 100 pfu, so the prime mission had a directive that commands be issued to place the spacecraft in a standby mode, powering off the 
instruments and common electronics. Two years into operation, a system-level analysis concluded there was no risk to the instruments if they remained powered on during a flare, so it was better for Spitzer operational efficiency to simply monitor the effects and possibly redo any observations ${ }^{12}$. Therefore, all systems that can by affected by a flare are monitored during any CME that can impact the spacecraft. For more information on space weather effects on systems other than the IRAC instrument, see Cheng et al 2014 for a full description.

\subsection{Effect of Space Weather on IRAC}

During the Warm and Beyond Missions, the IRAC instrument is operating both detector arrays full-time, and therefore it can be effected by space weather at anytime. Impacts from a shower of energetic particles can often manifest as extensive number of saturated pixels over the nominal amount. Saturated pixels on IRAC are most often caused by a simple over-exposure in the infrared of an astronomical source, not necessarily from space weather. In a saturated image, if the infrared flux on a single pixel is high enough, the readout of the entire column can be affected. This effect, called "column pulldown," is visible in on the left image in figure 5. In that figure, the central pixels from each of the two saturated stars have exceeded the flux threshold on the pixel, and therefore depressed the flux from all the pixels in the column. The pixel flux associated with the pulldown source can be modeled from the surrounding pixels, and therefore these astronomical based errors are easily correctable with standard data processing.
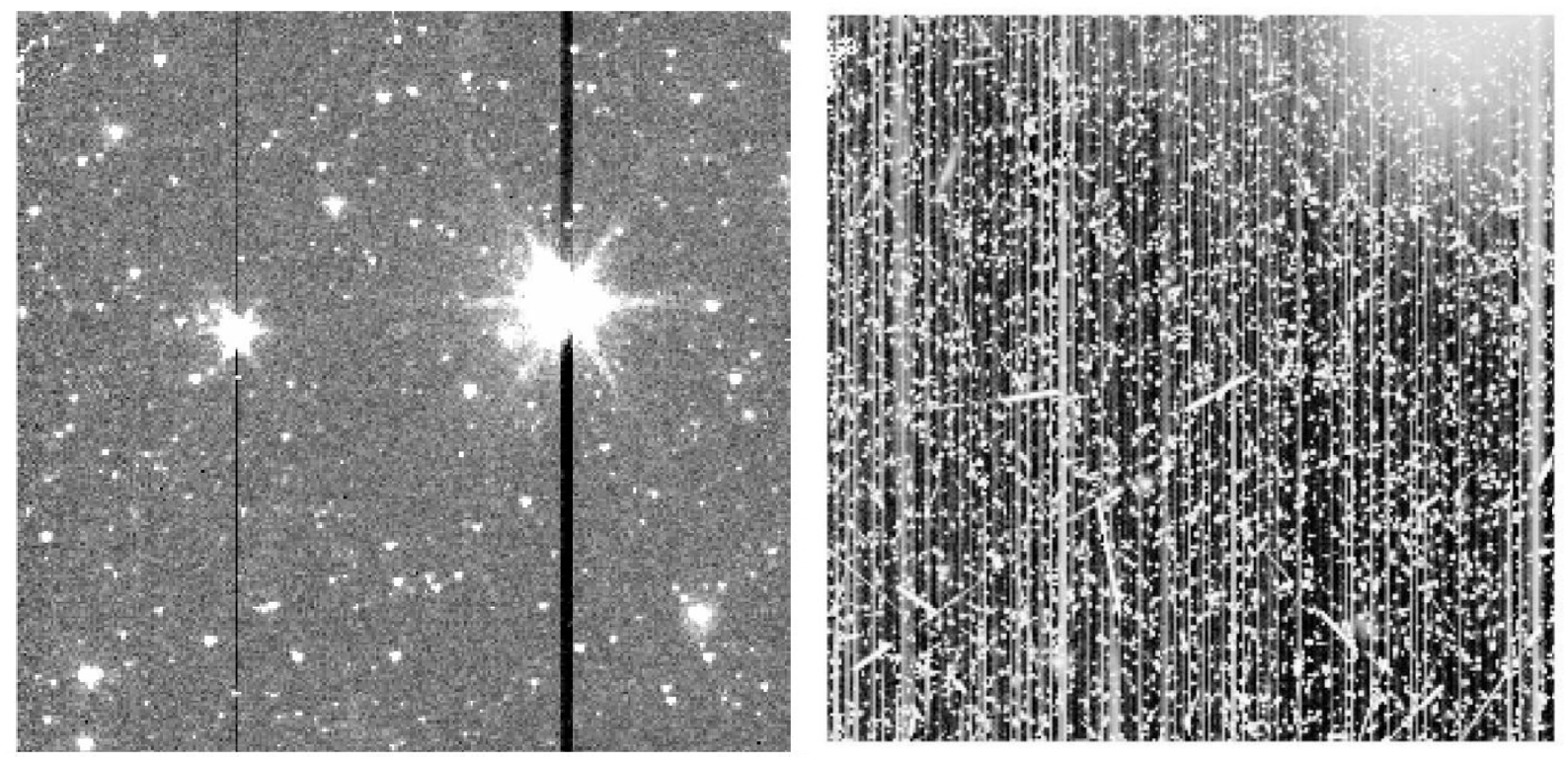

Figure 5. (left) Example of a 12 second observation taken with the 3.6 $\mu \mathrm{m}$ array The two brightest sources have large enough flux to cause a decrease in the flux along the entire readout column, called "column pulldown". (right) An observation of similar exposure time taken during an impact of a CME which displays a large number of streaks and column pulldown caused by high-energy particles.

As mentioned previously, in the absence of solar flares or CMEs, there are approximately 4-6 affected pixels per second from nominal extra-solar high-energy particles and cosmic rays. Occasionally, a normal cosmic ray will have enough energy to saturate a pixel on impact and cause the column pulldown effect to occur. Due to the inability to model the flux of the cosmic ray, these types of column pulldown cannot be corrected within the data reduction pipeline.

If a data frame is observed to have more than ten pixels affected by cosmic rays, the team investigates to determine a possible origin. There is no directional information for these particle hits, so correlating the timing with solar monitoring data helps to determine if the change is due to a possible solar flare. A shower of high-energy particles, triggered by a large solar flare or CME, can lead to a substantial number of affected pixels, including a large number of saturated pixels. The saturated pixels will cause whole columns to display the column pulldown effect, and the combination of these effects will render data frames unusable for science. 
For the example in Fig. 5b, none of the column pulldown effects were associated with saturated star images. This implies the majority of the affected pixels were caused by high-energy particle hits. Moreover, this precluded any useful astronomical observation, but resulted in no permanent damage to the instrument. In its lifetime, IRAC has experienced only a few large CME events while powered on, as mentioned earlier. One of the largest occurred after a series of flares in January $2005^{11}$. The CME events have a range of strength, but a handful of events have contained a high enough number of large energetic particles to cause disruption in the astronomical observations.

\subsection{IRAC Team's Response to Space Weather Events}

The IRAC instrument team closely monitors all solar flares to understand the overall effect before science data are released to the science investigator. Integration times for the full arrays can vary from 2 seconds to 100 seconds; therefore, single exposures number in the thousands per day. As checking every frame is cumbersome, an average of affected pixels is calculated over a single observational period pointed at one part of the sky. This usually consists of between 20 and 1000 single frames of the same exposure time. The data processing pipeline creates a mask file that keeps track of any problem pixels for every observation frame. Each frame is 256 by 256 pixels, and the mask is created with the same number of pixels, mirroring its associated observation frame. During processing, if a problem is found with a pixel or group of pixels in the observation, the same group in the pixel mask will be given a value associated with that problem. One such problem might be a cosmic ray, so an "outlier rejection" algorithm is used to compare multiple dithered frames stacked by astronomical, or sky, coordinates. As the telescope's viewpoint moves across the sky, an astronomical source will be observed in multiple pixels across the array, but a cosmic ray will only affect one pixel. When stacked by astronomical coordinates, the astronomical source should appear in multiple frames, so an "outlier" is a pixel with measurable flux only appearing in one of the frames. Those outlier pixels are then flagged as "radhits", marked in the pixel masks, and excluded when creating a mosaic, or image map, of the observations. This serves to create artifact-clean images while preserving the processing history.

During the Spitzer mission, the statistical baseline of average number of radhits per second on the IRAC array has been established as approximately 4 hits per second, mostly due to cosmic rays. It has been observed that a slightly higher number of cosmic rays (approximately 5 hits per second) is detected during solar minimum, and a lower number is detected during solar maximum (just over 3 per second).
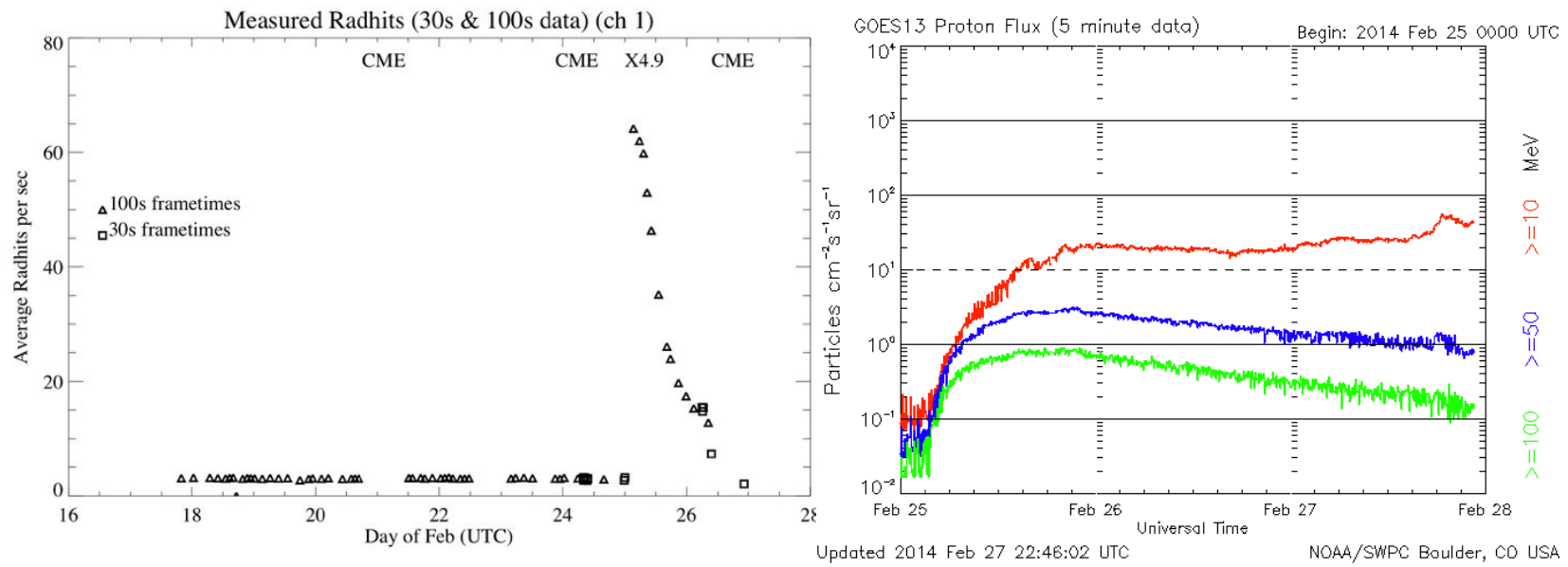

Figure 6. (left) The average number of radiation hits per second detected by the $3.6 \mu \mathrm{m}$ array during the ten days around the February 25, $2014 \mathrm{CME}$. The average radiation hits per second rose from a nominal 4 hits per sec to over 60 hits per sec and decreased over a 24 hour period. (right) The GOES 13 measure of the Proton flux over the period from the Feb 25, 2014 CME in which the IRAC arrays were affected.

Therefore, an IRAC team member receives alerts from the Goddard SWRC, which uses improved space weather models and includes Spitzer's ephemeris in their visualization tool. The alert specifies if Spitzer is predicted to be impacted. The 
masks from all the data for a week long period surrounding the predicted impact are then examined. Not all impacts produce a rise in radiation hits on IRAC, but a report is generated to add along with the other systems that are checked. As shown in Figure 6, during the February 2014 CMEs, the number of radiation hits per second measured by IRAC rose to over 60 shortly after predicted impact. The observations on IRAC are taken with a variety of exposure times, therefore the $12 \mathrm{~s}, 30 \mathrm{~s}$, and $100 \mathrm{~s}$ exposure time frame data are uses to gain a good statistical measure of the radiation hits per second. The GOES 13 never measured to $100 \mathrm{MeV}$ particles rise above 100 pfu and leveled off, while the number of radiation hits measured on IRAC fell quickly within the 24 hours after the maximum. This is most likely due to the different locations of the Spitzer and Earth orbits. As Spitzer has drifted farther away from Earth, GOES data are no longer directly indicative of the environment directly around the Spitzer spacecraft, but the inclusion of Spitzer ephemerides in the models has provided the information needed to directly monitor and trend the solar flare timing.

\section{HIGH PRECISION PHOTOMETRY}

Time series observations are used on IRAC to observe temporal variations in relative photometry on timescales from a few hours up to more than 20 days for exoplanets, brown dwarfs, and the Active Galactic Nuclei at the Galactic center. The first exoplanet observations employed a dithering strategy (intermittently shifting the position of a target on a detector array), but observers quickly discovered that the main systematic limiting the precision of photometry is changes in position coupled with intrapixel gain variations. The Spitzer Science Center now recommends continuous staring without internal re-pointing for any high precision time series observations. The source is placed on a wellcharacterized "sweet-spot" on a region of the least responsivity variation ${ }^{6}$. It was found the systematics in telescope motions and pointing could be characterized in short term drift, long term ( $>2 \mathrm{hr})$ drift, cloud size, and position oscillations which are trended using an archive of every staring observation ${ }^{8}$. Here we discuss two noted trends that are are correlated and seem to have changes associated with the increasing orbit of Spitzer.

\subsection{Pointing Oscillations}

The timescale of interesting features in exoplanet observations is of order one hour. Coincidentally, the battery heater originally cycled on roughly one-hour timescales as well. This heater cycling leads to thermal-mechanical variations, which cause $\sim 0.03$ " position changes of science targets on the IRAC detectors, or a "pointing wobble". Because the gain varies significantly (several percent compared to hundred parts per million fluctuations from exoplanets) as a function of position within a pixel, the resulting gain variations on timescales that are scientifically interesting can be a chief source of correlated noise, and thereby make it very difficult to disentangle science from instrument performance. In response, the Spitzer engineering team tightened the heater deadband so that it cycled on a period of approximately 40 minutes resulting in a pointing fluctuation of $<0.015$ arcsec. Separating the instrumental and planetary transit timescales and reducing the amplitude of fluctuations, led to increased photometric precision.

\subsection{Increasing Pitch Angles}

As the Spitzer mission traveled farther from the Earth in its orbit, the geometry of Spitzer, Earth and the Sun created a challenge. The pitch angle is the angle between the normal vector of the solar array and Sun direction vector as seen by Spitzer with zero degrees defined as solar panels directly facing the Sun. The spacecraft had to exceed a pitch angle of 30 degrees for downlinks in November 2013. In January 2016 the pitch angle of 40 degrees was exceeded. As the mission operations continue, there is a concern that as pitch angles increased, more area might be exposed to possible heating due to light falling on the spacecraft underneath the solar panel. This was coupled with the thermal and power constraints due to smaller percentage of illumination upon the solar panels, as well. In anticipation of ever increasing pitch angles, operational "toe-dips" were performed approximately 9-12 months before a milestone positon angle of $42.5,45.5$, and 48.5 degrees were reached, respectively. The spacecraft would pitch over at the desired angle and dwell for a given time to test battery usage and recharging. The spacecraft would then return to a pitch angle of 0 degrees to recharge the battery. The IRAC IST took advantage of these engineering tests and placed an observation directly after the engineering test that contained an 8-10 hour staring observation of a standard star. 

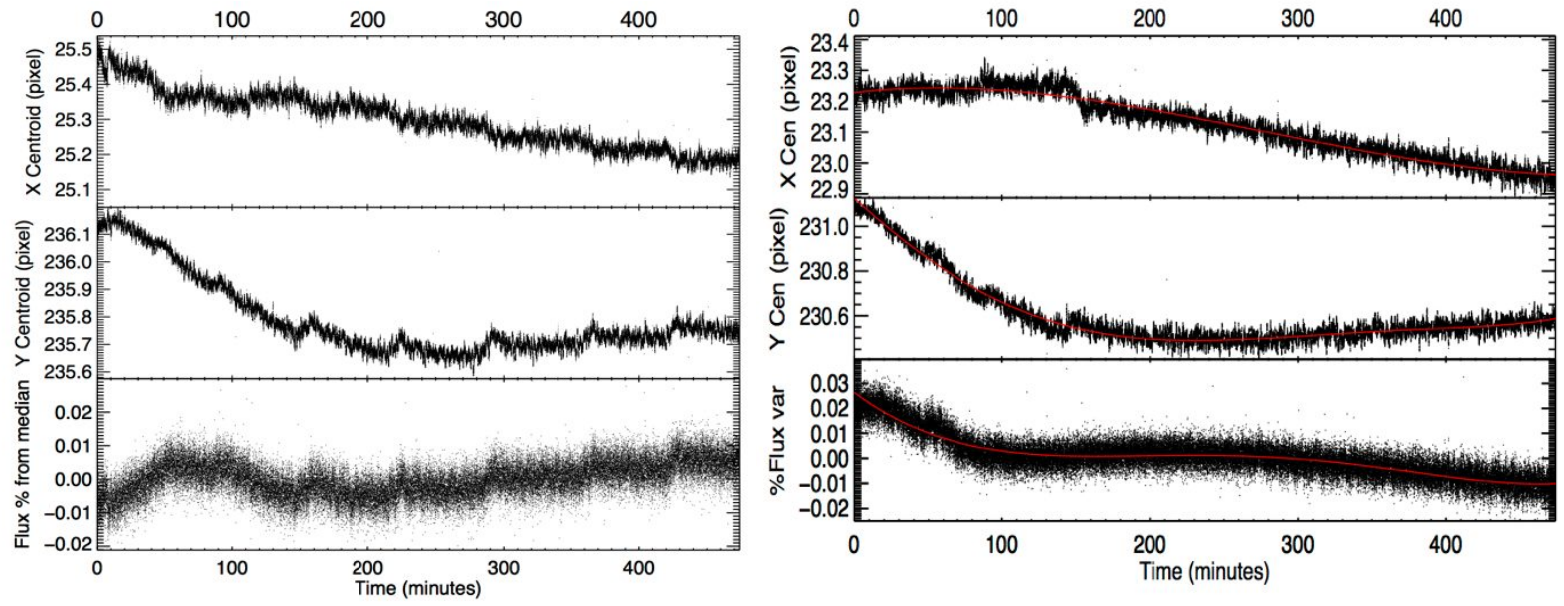

Figure 7. Staring Mode observations after a 3 hour "dwell" at pitch angles of 42.5 degrees and 45.5 degrees. The pointing wobble frequency is similar but more amplitude is decreased as the position angle increases. The redline in the 45.5 degree plot represents a polynomial fit to the data. The observations following the 48.5 degree dwell are not significantly different than the $\mathbf{4 5 . 5}$ degree observations. As of 2018, the pitch angle for downlinks is just less than 48 degrees.

The staring observation was analyzed to understand if any of the known systematics increased or changed (Figure 7). As the position angles increased, the IRAC IST found nothing that would hamper time series observations. It was observed that the short term drift (initial temperature settling of the telescope seen here in the first 2 hours) did seem to increase in timescale from a nominal value close to 30 minutes to about 2 hours, but the pointing oscillations have decreased. The source of the pointing oscillations had been the battery heater cycling. Telemetry was examined and found the heater was cycling less often due to the self-heating of a battery needed to be used more during the larger pitch angles. The short term drift increase is tied to the larger change in pitch angle (see [13]), and is in line with expectations based on previous observations.

\section{CONCLUSIONS}

The infrared observational capabilities of the Spitzer Space Telescope within the Warm and Beyond phases remain undiminished or improved despite the challenges presented by an aging instrument or those limitations encountered by an increasing distance from Earth. The stability of the Spitzer spacecraft and the IRAC instrument are a testament to the excellent design and engineering of the original teams. The sustained innovation and understanding of the calibrations and challenges have continued to open new avenues to produce bountiful science past the original design.

Proactive teams have anticipated the possible hazards that exist as the mission ages. As possible operational changes have been foreseen, all possible effects to science observations have been examined and often revisited. All options or changes can then be modeled thoroughly under differing conditions, therefore gaining a full understanding of all risks and gains. These can be implemented well in advance of problems or limitations to the mission. The different ways Spitzer has optimized its existing capabilities and the associated lessons learned that have gone into maximizing the lifetime well into its second decade of operation will hopefully provide guidelines for future missions, as it continues to make important contributions to the field of astrophysics. 


\section{REFERENCES}

[1] Werner, M. et al., "The Spitzer Space Telescope Mission," Astrophysical Journal Supplement Series, Volume 154, Issue 1, 2004, pp. 10-17. doi: 10.1086/422992

[2] Fazio, G.G. et al., "The Infrared Array Camera (IRAC) for the Spitzer Space Telescope," Astrophysical Journal Supplement Series, Volume 154, Issue 1, 2004, pp. 10-17, doi: 10.1086/422843

[3] http://ssc.spitzer.caltech.edu/warmmission/scheduling/iracCalTrending/

[4] Reach et al., "Absolute Calibration of the Infrared Camera on the Spitzer Space Telescope", Publications of the Astronomy Society of the Pacific, Volume 117, Issue 835, 2005, pp978, doi: 10.1086/432670

[5] Carey, S. et al. "Calibration and data quality of Warm IRAC", Proc. Volume 7731, Space Telescopes and Instrumentation 2010: Optical, Infrared, and Millimeter Wave; 77310N (2010) https://doi.org/10.1117/12.857788

[6] Ingalls, James G. et al "Repeatability ad Accuracy of Exoplanet Eclipse Depths Measured with Post-cyrogenic Spitzer", Astronomical Journal, Volume 152, Issue 2, 2016, pp. 44-71, doi: 10.3847/0004-6256/152/2/44

[7] Ingalls, James G. et al. "Intra-pixel gain variations and high precision photometry with the Infrared Array Camera (IRAC)", Proc. Volume 8442, Space Telescopes and Instrumentation 2012: Optical, Infrared, and Millimeter Wave; 84421Y (2012) https://doi.org/10.1117/12.926947

[8] Grillmair, Carl J. et al. "Pointing Effects and their consequences for Spitzer IRAC exoplanet observations", Proc. Volume 8448, Observatory Operations: Strategies, Processes, and Systems IV; 84481I (2012) https://doi.org/10.1117/12.927191

[9] Krick, J. et al. "A Spitzer IRAC measure of zodiacal light", Proc. Volume 8442, Space Telescopes and Instrumentation 2012: Optical, Infrared, and Millimeter Wave; 84421X (2012) https://doi.org/10.1117/12.926664

[10] Gosling, J. T., "The Solar Wind," Encyclopedia of the Solar System, 2nd ed., edited by L. McFadden, P.R. Weisman, and T.V. Johnson, Academic Press, San Diego, CA, 2007, pp. 99-116.

[11] Hora, J. L., Patten, B. M., Fazio, G. G., and Glaccum, W.J., "The Effects of Cosmic Rays and Solar Flares on IRAC Detectors" the First Two Years of In-flight Operation" Proc. SPIE, Vol. 6276, 2006, pp. 62760J1-15

[12]Chung, Leo Y., "Investigating Space Weather Events Impacting the Spitzer Space Telescope", May 2014; 13th International Conference on Space Operations (SpaceOps 2014), doi: 10.2514/6.2014-1910

[13] Krick, J. et al. "Using the Science Archive for Instrument Trending on Spitzer IRAC", Proceedings SPIE, this issue 